\title{
ARTICLE
}

\section{Disruption of water networks is the cause of human/mouse species selectivity in urokinase plasminogen activator (UPA) inhibitors derived from hexamethylene amiloride (HMA)}

Received 00th January 20xx, Accepted 00th January 20xx DOI: $10.1039 / \times 0 \times x 00000 x$
Nehad S El Salamouni, ${ }^{a, b, c}$ Benjamin J Buckley, ${ }^{a, b, c, d}$ Longguang Jiang, e Mingdong Huang, ${ }^{e}$ Marie Ranson, ${ }^{a, b, c, d}$ Michael J Kelso, ${ }^{a, b, c, *}$ and Haibo $\mathrm{Yu}^{\mathrm{a}, \mathrm{b}, \mathrm{c},{ }^{*}}$

\begin{abstract}
The serine protease urokinase plasminogen activator (UPA) plays a critical role in tumour cell invasion and migration and is a promising anti-metastasis drug target. 6-Substituted analogues of 5- $\mathrm{N}, \mathrm{N}$-(hexamethylene)amiloride (HMA) are potent uPA inhibitors that show anti-metastatic effects in vivo and high selectivity over closely related trypsin-like serine proteases, while lacking the diuretic and anti-kaliuretic properties of the parent drug amiloride. However, the compounds as a class display pronounced selectivity for human over mouse uPA, thus confounding interpretation of data from human xenografted mouse models of cancer. To understand the molecular basis of this selectivity, we performed molecular dynamics simulations and alchemical free energy perturbation calculations using human and mouse uPA and their complexes with amiloride, HMA and 6-substituted HMA analogues and compared the results to enzyme inhibitory potencies. X-ray structures of selected compounds bound to partially murinised human uPA (H99Y) were also obtained. Collectively, the computational and experimental findings revealed that residue 99 is a key contributor to human/mouse uPA species selectivity, whereby enthalpically unfavourable steric expulsion of a water molecule by the 5- $N, N$-hexamethylene ring of HMA and analogues occurs when residue 99 is Tyr (as in mouse uPA). Analogue 7 lacking the 5-N,N-hexamethylene ring maintained similar water networks when bound to human and mouse uPA and displayed reduced selectivity, thus supporting this conclusion. The study highlights the crucial role water molecules can play in protein ligand binding and will serve to guide further optimisation of dual-potent human/mouse uPA inhibitors from the amiloride class as anti-metastasis drugs.
\end{abstract}

\section{Introduction}

The urokinase plasminogen activation system (UPAS) comprises the trypsin-like serine protease (TLSP) urokinase plasminogen activator (UPA), its cognate cell surface receptor (UPAR) and three endogenous serpin inhibitors; plasminogen activator inhibitors PAI-1, PAI-2 and PAI-3.1-5 A major function of UPA is to catalyse the conversion of plasminogen to plasmin, which in turn activates multiple downstream proteases, including matrix metalloproteinases (MMPs) and cathepsins. ${ }^{6}$ Collectively, these activated proteases cause localised proteolysis that leads to basement membrane degradation and remodelling of the extracellular matrix. ${ }^{4,5}$ As such, the uPAS is intimately involved in several physiological functions that require controlled tissue remodelling, including ovulation, 7, 8 embryonic implantation, ${ }^{9}$

\footnotetext{
a. School of Chemistry and Molecular Bioscience, University of Wollongong, NSW 2522, Australia.mkelso@uow.edu.au (MK), hyu@uow.edu.au (HY)

b. Molecular Horizons, University of Wollongong, NSW 2522, Australia.

c. Illawarra Health and Medical Research Institute, Wollongong, NSW 2522, Australia.

d. CONCERT-Translational Cancer Research Centre, NSW 2750, Australia.

e. National Joint Biomedical Engineering Research Centre on Photodynamic Technologies, Fuzhou University, Fujian 350116, China.

+ Electronic Supplementary Information (ESI) available: Supplementary Fig. S1-S18 and Tables S1-S17. See DOI: 10.1039/x0xx00000x
}

mammary gland involution, 10 inflammatory reactions, ${ }^{11}$ wound healing ${ }^{11}$ and clot lysis. ${ }^{12,13}$ Dysregulated uPA activity causes tissue damage that has been linked to rheumatoid arthritis, 14,15 allergic vasculitis, ${ }^{16}$ xeroderma pigmentosum, ${ }^{17}$ multiple sclerosis ${ }^{18}$, lymphangioleiomyomatosis, ${ }^{19}$ and chronic kidney disease. ${ }^{20}$ Aberrant uPA activity is also implicated in tumour cell growth, migration, invasion and metastasis, where it contributes to poor prognosis in multiple cancer types ${ }^{21}$ (e.g. gastric, ${ }^{22}$ gastroesophageal, ${ }^{23}$ ovarian, ${ }^{24}$ pancreatic ${ }^{25}$ and breast ${ }^{26}$ ). In node-negative breast cancer, primary tumour uPA levels are a reliable prognostic marker of poor patient outcomes.27, 28 Several studies have revealed that pharmacological inhibition of UPA can reduce tumour growth and metastasis in rodent models, 1, 29, 30 supporting UPA as an anticancer/metastasis drug target.

uPA present in the tumour stoma plays a central role in cell growth and dissemination in xenograft rodent models. ${ }^{31}$ Depending on the tumour type, host stromal cells (e.g. endothelial cells, fibroblasts and macrophages) can be the predominant uPA-expressing tissue. ${ }^{32}$ Human breast xenograft models in mice have demonstrated that stroma expressed mouse UPA binds to cell-surface UPAR in the engrafted tumour tissue, albeit with lower affinity relative to the endogenous human UPA-UPAR interaction. ${ }^{33-35}$ Thus, uPA-targeting anticancer drugs presumably must inhibit UPA from both the 
tumour and associated host stromal cells for maximal effect in xenograft models. ${ }^{36}$

The oral $\mathrm{K}^{+}$-sparing diuretic amiloride has repeatedly been shown to exhibit anticancer side-activities in biochemical and rodent models. ${ }^{37}$ These activities appear to arise, at least in part, from moderate inhibition of uPA $\left(K_{\mathrm{i}}=7 \mu \mathrm{M}\right) .37,38$ The reported $\mathrm{X}$-ray co-crystal structure of amiloride bound to human uPA (PDB 1F5L; $2.1 \AA \AA^{39}$ identified that amiloride occupies the active site of the protease, burying its acylguanidine deep into the S1 specificity pocket to form a critical salt bridge interaction with the side chain carboxylate of Asp189. Many other polar interactions are also present (Fig. S1). Amiloride's interactions with uPA have also been modelled. 40

We recently reported on the structure-activity relationships around UPA inhibition by 6-substituted analogues of amiloride ${ }^{30}$ and $5-N, N$-(hexamethylene)amiloride (HMA), ${ }^{41}$ a well-studied derivative with similar in vitro and in vivo anticancer properties. ${ }^{42-44}$ Several analogues were identified that showed significantly enhanced potency (relative to amiloride and HMA) against human UPA ( $\mathrm{IC}_{50}$ low nanomolar range) and lead compounds demonstrated anti-metastatic properties in mouse xenograft models. An X-ray co-crystal structure of HMA bound to human uPA (PDB 5ZA7; $1.7 \AA)^{41}$ confirmed that the key salt bridge interaction between the acylguanidine and Asp189 is maintained upon introduction of the 5- $N, N$-hexamethylene ring at the 5-position of amiloride, as are most of the other polar contacts (Fig. S2). X-ray co-crystal structures of 6-substituted HMA derivatives revealed similar binding orientations and hydrogen bonding patterns as amiloride and HMA. ${ }^{41}$ Importantly, the newly appended substituents at the 6-position were all oriented towards the $S 1 \beta$ subsite. We concluded that the increased UPA inhibitory potency of 6-substituted HMA analogues (relative to amiloride and HMA) was due to favourable new contacts formed between the 6 -substituents and the S1 $\beta$ subsite. ${ }^{37,41}$ This conclusion aligned with earlier reports on 4-amidinobenzothiophene and 8-naphthamidine-based inhibitors that target the S1 $\beta$ site. ${ }^{45}$ In the amiloride (Fig. S1), ${ }^{39}$ HMA (Fig. S2) ${ }^{41}$ and 6-substituted HMA human uPA X-ray co-crystal structures (PDB 5ZA9; $1.62 \AA$, PDB 5ZAH; $2.98 \AA$ and PDB 5ZAJ; $1.65 \AA$ ),, ${ }^{41}$ a highly conserved water molecule bound in the $S 1$ pocket forms a hydrogen bonding network between the carbonyl oxygen of the acylguanidine in the ligands and the backbone amide nitrogen of Ser214 and the backbone carbonyl oxygen of Val227. Ligands also form hydrogen bonds with the side chain hydroxyl of Ser190. Hydrogen bond interactions of this type are known to impart selectivity for TLSPs that contain Ser190 (e.g. UPA, trypsin, FVIla) over relatives that feature Ala at this position (e.g. tPA, thrombin, FXa). ${ }^{46}$ Selectivity over the Ala 190 subgroup is increased for inhibitors that displace the highly conserved S1 water molecule. ${ }^{47,48}$

The human and mouse uPA enzymes show $71 \%$ homology in their protease domains, ${ }^{49}$ with their active sites differing at only four residues; human uPA: Asp60, His99, Ser146 and
GIn192; mouse uPA: GIn60, Tyr99, Glu146 and Lys192 (Fig. S3)..$^{49}$ Despite this apparently small difference, small molecule active site inhibitors of UPA often show a pronounced species preference for human over mouse uPA. ${ }^{41}$, 49 Interestingly, amiloride 1 is one of the few inhibitors that show comparable potency against both enzymes (human/mouse selectivity ratio: 0.9, Table 1). ${ }^{41,49}$ We found that introduction of the $5-\mathrm{N}, \mathrm{N}-$ hexamethylene ring at the 5-position of amiloride 1 (e.g. HMA 2) reduces mouse uPA potency (human/mouse selectivity ratio: 6.9) and that this bias is accentuated in 6-substituted HMA analogues (e.g. compounds 3-6, Table 1), where selectivity for the human enzyme can exceed 130 -fold. ${ }^{41}$ Such large species differences complicate the development of UPA inhibitors as the unknown effects of poorly inhibited murine stromal uPA confound interpretation of on-target effects in human-mouse xenograft tumour models. ${ }^{50}$ Compounds that show similar potency against human and mouse uPA would provide greater confidence that observed efficacy arises from uPA inhibition.

In this study, molecular dynamics (MD) simulations coupled with alchemical free energy perturbation (FEP) calculations were used to probe the structural basis of the species differences observed for HMA 2 and 6-substituted analogues 36 against human and mouse uPA. Biochemical enzyme assays and X-ray co-crystal structures of selected analogues bound to partially murinised human uPA (H99Y) were used to support the findings.

\section{Experimental}

\section{Molecular docking}

Docking was performed using Autodock Vina 1.1.2.51 Structures were first prepared and optimised using ChemDraw 16.0 and Avogadro 1.2.0.52 The acylguanidine was protonated for all ligands. AutoDockTools 1.5.653 was used to assign rigid and rotatable bonds and to remove non-polar hydrogens. Docking was performed in a 22 $\AA \times 22 \AA \times 22 \AA$ box centred at the active site of uPA.

\section{Molecular dynamics (MD) simulations}

MD simulations were carried out using NAMD 2.12.54 Initial preparation of systems was performed using VMD 1.9.2. ${ }^{55}$ The AMBER parm14SB force field 56,57 was applied for the protein and the TIP3P model was used for water. ${ }^{58}$ The general Amber Force Field $(\mathrm{GAFF})^{59}$, 60 was used for parameterisation of inhibitors using the Antechamber package, with partial charges assigned using the AM1-BCC scheme. ${ }^{61}$ The GAFF-based force field parameters for ligands 1-7 can be accessed from doi.org/10.6084/m9.figshare.14446440.v1. MD simulations of the complexes were performed after solvating the system in an $84 \AA \times 84 \AA$ × $84 \AA$ cubic TIP3P water box that extended at least $10 \AA$ from the protein surface. $\mathrm{Na}^{+}$and $\mathrm{Cl}^{-}$counter ions were added to neutralise the system and achieve a salt concentration of $0.15 \mathrm{M}$. 
Table 1 Inhibitory potencies of amiloride 1, HMA 2 and 6-substituted analogues 3-6 against human and mouse uPA.

\begin{tabular}{|c|c|c|c|c|}
\hline Ligands & Structure & $\begin{array}{c}\text { Human uPA } \\
K_{\mathrm{i}}(\mathrm{nM})\end{array}$ & $\begin{array}{c}\text { Mouse uPA } \\
K_{\mathrm{i}}(\mathrm{nM})^{b}\end{array}$ & $\begin{array}{l}\text { Human/Mouse } \\
\text { selectivity factor }\end{array}$ \\
\hline Amiloride 1 & & $2,433 \pm 192$ & 2,313 & 0.9 \\
\hline HMA 2 & & $1,356 \pm 183$ & 9,308 & 6.9 \\
\hline 3 & & $183 \pm 10$ & 1,802 & 9.9 \\
\hline & & $53 \pm 5$ & 1,611 & 30.4 \\
\hline 5 & & $42 \pm 2$ & $3,293^{a}$ & 78.4 \\
\hline 6 & & $21 \pm 3$ & $2,768^{a}$ & 131.8 \\
\hline
\end{tabular}

${ }^{a}$ Measured in this work. All other data reported previously. ${ }^{41}$

${ }^{b}$ Values represent the average of two independent experiments. ${ }^{41}$

$\mathrm{p} K_{\mathrm{a}}$ calculations were performed on the protein-ligand complexes using PROPKA 62 to assign protonation states of ionisable residues. Simulations were performed using periodic boundary conditions (PBC) at constant temperature $(298.15 \mathrm{~K})$ with the Langevin algorithm at a pressure of 1.0 bar using the Nose-Hoover Langevin Piston method (time step $=2.0 \mathrm{fs}$ ) ${ }^{63}$ All covalent bonds involving hydrogens were kept rigid with the RATTLE algorithm. ${ }^{64}$ The Particle Mesh Ewald (PME) algorithm ${ }^{65}$ was applied for long-range electrostatic interactions with a $10 \AA$ A distance cut off. For all systems, energy minimisation and $1.0 \mathrm{~ns}$ equilibration were performed first with positional restraints placed on heavy atoms. This was followed by $100 \mathrm{~ns}$ production runs. All simulations were performed in triplicate with different initial velocities and snapshots were saved every 500 steps (1.0 ps). VMD 1.9.2 was used for visualisation of trajectories and analysis of simulations. ${ }^{55}$ Atomic positional rootmean-square deviations (RMSD) and atomic positional root-meansquare fluctuations (RMSF) with respect to starting structures were calculated to monitor global properties of the structural evolution during simulations. RMSF of the protein a-carbon $\left(C_{a}\right)$ atoms of the $\mathrm{X}$-ray crystal structures were calculated from the $\mathrm{C}_{\mathrm{a}} \mathrm{B}$-factors using the equation: $R M S F=\sqrt{3 B / 8 \pi^{2}} .66$ Hydrogen bond interactions from $\mathrm{MD}$ trajectories were analysed using $\mathrm{HBonanza}^{67}$ using a 
hydrogen-donor-acceptor angle cutoff of $30^{\circ}$ and distance cutoff of $3.0 \AA$ A. Pairwise interaction energy analysis was performed on MD simulation trajectories using the NAMD Energy Plugin 1.4 of VMD. ${ }^{54}$ Average water density maps of the combined triplicate MD simulations were calculated using the Volmap plugin in VMD. ${ }^{55} \mathrm{~A}$ summary of all simulated systems is provided in Table S1.

\section{Alchemical free energy perturbation calculations}

Free energy differences for the binding of ligands to proteins were calculated using the thermodynamic cycle outlined in Fig. S4. The binding free energy measured experimentally is represented by the horizontal legs. The vertical legs represent the alchemical transformation by computer simulations, in solution $\left(\Delta \mathbf{G}_{\mathrm{aq}}^{\mathbf{L i g A} \rightarrow \mathbf{L i g B}}\right)$ and in the protein-ligand complex $\left(\Delta \mathbf{G}_{\text {complex }}^{\mathbf{L i g A} \rightarrow \text { LigB }}\right)$. Relative binding free energy differences between ligand $A$ and ligand $B$ were calculated using Eq. 1

$$
\underset{\text { (Eq. 1) }}{\Delta \Delta \mathbf{G}_{\mathbf{b}}^{\text {LigA } \rightarrow \text { LigB }}}=\Delta \mathbf{G}_{\mathbf{b}}^{\text {LigB }}-\mathbf{G}_{\mathbf{b}}^{\text {LigA }}=\Delta \mathbf{G}_{\text {complex }}^{\text {LigA } \rightarrow \text { LigB }}-\Delta \mathbf{G}_{\mathrm{aq}}^{\text {LigA } \rightarrow \text { LigB }}
$$

Relative binding free energy differences for ligands binding to uPA and partially murinised uPA (uPA ${ }^{\mathrm{m}}$ ) were calculated using Eq. 2 $\Delta \Delta \mathrm{G}_{\mathrm{b}}^{\mathrm{uPA} \rightarrow \mathrm{uPA}^{\mathrm{m}}}=\Delta \mathrm{G}_{\mathrm{b}}^{\mathrm{uPA}}{ }^{\mathrm{m}}-\Delta \mathrm{G}_{\mathrm{b}}^{\mathrm{uPA}}=\Delta \mathrm{G}_{\text {complex }}^{\mathrm{uPA} \rightarrow \mathrm{uPA}^{\mathrm{m}}}-\Delta \mathrm{G}_{\mathrm{aq}}^{\mathrm{uPA} \rightarrow \mathrm{uPA}^{\mathrm{m}}}$ (Eq. 2)

$\mathrm{G}_{\text {complex }}^{\mathrm{LigA} \rightarrow \text { LigB }}, \Delta \mathrm{G}_{\mathrm{aq}}^{\mathrm{LigA} \rightarrow \text { LigB }}, \Delta \mathrm{G}_{\text {complex }}^{\mathrm{uPA} \rightarrow \mathrm{uPA}^{\mathrm{m}}}$ and $\Delta \mathrm{G}_{\mathrm{aq}}^{\mathrm{uPA} \rightarrow \mathrm{uPA}^{\mathrm{m}}}$ were calculated using the alchemical free energy perturbation (FEP) simulations. ${ }^{68,} 69$ All FEP simulations were performed using NAMD 2.12. ${ }^{54}$ Alchemical transformations of ligands and protein residues were performed with the dual topology scheme using 20 equally distributed $\lambda$ windows between 0 and $1(0.0,0.05,0.1, \ldots, 0.9,0.95$, 1.0), bidirectionally (i.e. forward and backward simulations). To prevent numerical instabilities as atoms were created or destroyed a soft-core potential was used with alchVdWShiftCoeff $=4.0 .{ }^{70} \mathrm{Initial}$ 1.0 ns equilibration simulations were carried out for each system, including 1,000 minimisation steps followed by multiple nanoseconds of production runs. In FEP calculations involving perturbation of ligands, a weak harmonic restraint was placed on similar heavy atoms with a force constant of $1.0 \mathrm{kcal} \mathrm{mol}^{-1} \AA^{-2}$ to address the wandering-ligand problem. ${ }^{71}$ ParseFep plugin $2.0^{72}$ within VMD 1.9.2 was used to compute the free energy differences as well as estimate the statistical error where the forward and backward simulations were combined using the Bennett acceptance ratio (BAR) estimator. ${ }^{73}$ There was no net charge in the FEP and it was expected that the correction for electrostatic artefacts were negligible. An equation analogous to the van't Hoff equation was used to decompose free energy to its enthalpic and entropic components. ${ }^{74,75}$

\section{uPA Activity Assays}

Mouse uPA activity assays for compounds 5-7 were performed using active high molecular weight mouse urokinase, (MUPA, Molecular Innovations Inc., MI, USA) at a final enzyme concentration of $20 \mathrm{nM}$, as previously reported..$^{41}$ For ligands $\mathbf{1 - 3}$ and $\mathbf{5}$, inhibition of partially murinised human uPA (H99Y, $\left.{ }^{6} 2 \mathrm{nM}\right)$ activity was determined using the chromogenic substrate S-2444 (pyroGlu-Gly-Arg-pNA) at a final concentration of $250 \mu \mathrm{M}$ in $20 \mathrm{mM}$ HEPES, $100 \mathrm{mM} \mathrm{NaCl}, 0.5 \mathrm{mM}$ EDTA, $0.01 \%(v / v)$ Tween-20, $p H$ 7.6. Thawed enzyme stocks were maintained at $-20^{\circ} \mathrm{C}$. DMSO was present at a final concentration of $1 \% \mathrm{v} / \mathrm{v}$. Change in absorbance over time at $405 \mathrm{~nm}$ was measured at $37{ }^{\circ} \mathrm{C}$ using a BioTek Synergy 4 384-well plate reader (BioTek Instruments Inc., Winooski, Vermont, USA). $K_{\mathrm{i}}$ values were calculated using the method of Cheng and Prussof. ${ }^{77}$

\section{Crystallisation and X-ray Data Collection}

Expression and purification of partially murinised human uPA (H99Y) protease domain were performed as previously described. ${ }^{78}$ Crystals were grown by the sitting drop vapour diffusion method and were obtained by equilibrating against a reservoir solution containing 50 $\mathrm{mM}$ sodium citrate $(\mathrm{pH} 4.6)$ and $2.0 \mathrm{M}$ ammonium sulphate supplemented with 5\% PEG400 at room temperature for 3 days. For H99Y-inhibitor complexes, the crystals were soaked for 1 week in a new soaking buffer (40\% PEG4000, $100 \mathrm{mM}$ Tris- $\mathrm{HCl}, \mathrm{pH}$ 7.4) containing $100 \mu \mathrm{M}$ inhibitor. Prior to X-ray data collection, crystals were soaked in a cryoprotectant solution containing $20 \%$ glycerol and snap-frozen in liquid $\mathrm{N}_{2}$. X-ray diffraction data of the crystals were collected at the BL17U beamline, Shanghai Synchrotron Radiation Facility (SSRF). The diffraction data were indexed and integrated using the HKL2000 program package. ${ }^{79}$ Structures were solved by molecular replacement ${ }^{80}$ using the uPA structure (PDB 4DVA; $1.94 \AA)^{81}$ as the search model. Electron density of ligands was clearly visible in the H99Y active sites and the structure of the ligand was modelled based on the $F_{\mathrm{o}}-F_{\mathrm{c}}$ difference map. The structures were refined using the CCP4 program package ${ }^{79}$ and manually adjusted iteratively until convergence using the molecular graphics program COOT $^{82}$. Solvent molecules were added using a $F_{\mathrm{o}}-F_{\mathrm{c}}$ Fourier difference map at $2.5 \sigma$ in the final refinement step. Statistics for data collection and final model refinement are summarised in Table S2.

Coordinates of the partially murinised human UPA (H99Y) inhibitor complexes H99Y-2 (PDB 6JYP; $2.25 \AA$ )), H99Y-3 (PDB 6L05; $2.90 \AA$ ) and H99Y-5 (PDB 6L04; $2.40 \AA$ ) were deposited in the RSCB Protein Data Bank (PDB; www.rcsb.org).

\section{Results and discussion}

An initial requirement for the study was to identify a computational method that could recapitulate: (1) ligand poses observed in reported human ligand-uPA X-ray co-crystal structures and (2) experimentally determined human uPA inhibitory potencies. Validation of the method in this way was necessary before applying the approach to studies of ligand-bound mouse uPA complexes where no X-ray co-crystal structures existed.

\section{Molecular docking fails to predict human/mouse uPA activity}

Molecular docking using Autodock Vina ${ }^{51}$ was the first technique explored. X-ray co-crystal structures of the following complexes were used: human uPA-amiloride (huPA-1, PDB 1F5L; $2.1 \AA$ ) ), ${ }^{39}$ huPAHMA (huPA-2, PDB 5ZA7; $1.7 \AA$ Å), ${ }^{41}$ huPA-3 (PDB 5ZA9; $1.62 \AA$ ), ${ }^{41}$ huPA-4 (PDB 5ZAH; $2.98 \AA$ ), ${ }^{41}$ huPA-5 (PDB 5ZAJ; $1.65 \AA$ ). ${ }^{41}$ As an Xray structure was not available for the huPA- 6 complex, the docked structure of $\mathbf{6}$ was instead compared to the X-ray structure of its closely-related analogue $4 .{ }^{41}$ Ligands were removed from the human 
UPA X-ray co-crystal structures and docked back into the protein. In all cases, the position of the docked ligand matched the respective X-ray structure (Fig. S5). Although the docking protocol reproduced the correct binding poses, the rank order of ligand potencies inferred from the calculated binding free energies (Table S3; $\mathbf{3}>\mathbf{6}>$ amiloride $\mathbf{1}>\mathbf{4}>\mathbf{5}>$ HMA 2) did not match the order determined experimentally from enzyme assays (Table 1; $\mathbf{6}>\mathbf{5}>\mathbf{4}>\mathbf{3}>$ HMA 2 $>$ amiloride 1). Nevertheless, the protocol was used to dock amiloride 1, HMA 2 and analogues 3-6 into the reported X-ray structure of mouse uPA (PDB 5LHQ; $2.6 \AA$ $^{83}$ (Fig. S6). All ligands were found to bind with their acylguanidine interacting with Asp189. With the exception of $\mathbf{3}$, the ligands presented similar orientations of the pyrazine core and extended their 6 -substituents into the $S 1 \beta$ subsite. Despite producing sensible docking poses, the rank order of ligand potencies inferred from the binding free energies (Table S4; 3 $>\mathbf{6}>$ amiloride $\mathbf{1}>\mathbf{5}>\mathbf{4}>$ HMA 2 ) did not match the experimentally determined potencies (Table 1; $\mathbf{4}>\mathbf{3}>$ amiloride $\mathbf{1}>\mathbf{6}>\mathbf{5}>$ HMA 2). The lack of protein flexibility, solvent effects, inaccuracy of the Vina scoring function and the relatively small differences in activity between the inhibitors were possible reasons for the discrepancies. ${ }^{51}$ The inability of docking to faithfully capture the experimentally determined potencies against either human or mouse uPA shifted our focus to other modelling techniques.

\section{MD simulations reveal similar hydrogen bond interactions for} ligands binding to human and mouse uPA

To consider protein flexibility and evaluate the stability of proteinligand complexes, we performed MD simulations on apo-human and mouse UPA and their respective complexes with amiloride 1, HMA 2 and analogues 3-6. The X-ray structures of human UPA (PDB 1F5L; $2.1 \AA)^{39}$ and mouse uPA (PDB 5LHQ; $\left.2.6 \AA\right)^{83}$ were used as starting structures for simulations of the apo-proteins and $X$-ray structures of huPA-1, huPA-2, huPA-3, huPA-4 and huPA-5 were used for the huPA-ligand complexes. The starting structure for simulation of the huPA-6 complex was created by substituting the 4-methoxy group of the 6-(4-methoxypyrimidinyl) analogue (ligand 4) (PDB 5ZAH; 2.98 $\AA)^{41}$ with an amino group. Starting structures for all mouse uPAligand complexes corresponded to the docking poses described above.

To evaluate the stability of complexes throughout simulations, the RMSD values of the apo and ligand-bound protein $C_{a}$ atoms were monitored with respect to initial positions (Fig. S7). All structures were confirmed to be stable, producing variations of 1.0-2.5. . In general, higher RMSD values were observed in the mouse compared to the human UPA structures in both the apo and ligand-bound states. To explore the stability of ligands 1-6 in their complexes, RMSD values of ligand heavy atoms relative to starting structures were monitored throughout simulations (Fig. S8). The plots showed that ligands 1-6 were all tightly bound and higher RMSD values were observed in the mouse compared to the human uPA complexes.

To identify regions of flexibility in human and mouse uPA and in the ligand-bound complexes, the root-mean-square fluctuation (RMSF) of the protein $\mathrm{C}_{\mathrm{a}}$ atoms was monitored during simulations
(Fig. S9). RMSF of the $C_{a}$ atoms for the available human X-ray crystal structures calculated from the crystallographic B-factors was also compared to the RMSF values calculated from MD simulations. Regions of protein flexibility and rigidity were consistent between the RMSF derived from the X-ray B-factors and MD simulations. RMSF plots showed that mouse uPA displays higher fluctuations in several regions (Phe21 to Gln27, Val66 to Lys82, Gly142 to Asn154 and Ser214 to Val227) and higher overall flexibility than human uPA in both the apo and ligand-bound states (Fig. S9). Comparing the RMSF plots of both proteins did not reveal a difference in the dynamic behaviour of residues 60, 99, 146 and 192; the four binding site residues that differ between the human and mouse proteins or other parts of the proteins that might explain the human/mouse UPA selectivity of the ligands.

Hydrogen bond analysis was used to compare the ligand binding modes in their complexes with human and mouse uPA (Figs. 1, S10 and S11). In the human uPA complexes, ligands 1-6 maintained the salt bridge interaction between the acylguanidine and the Asp189 side chain carboxylate at the base of the S1 pocket. The hydrogen bonds between the terminal nitrogens of the acylguanidine and side chain hydroxyl of Ser190 and backbone carbonyl of Gly219, which were observed in the X-ray structures, were all maintained throughout the MD simulations. Ligand $\mathbf{4}$ showed additional hydrogen bonding interactions between the nitrogen (N1) of its acylguanidine and the backbone carbonyls of S1 $\beta$ residues Lys 224 and Arg217 (Table S5). The X-ray co-crystal structures of ligands 1-5 and docked structure of ligand $\mathbf{6}$ showed hydrogen bond interactions between the amino group at the pyrazine 3-position and the side chain hydroxyl of the catalytic Ser195 residue (Table S5). This hydrogen bond was maintained throughout the MD simulations of the human UPA complexes for the two closely related 6-(2substituted pyrimidine) analogues (ligands 4 and 6), only. This is likely due to ligand $\mathbf{6}$, and to a lesser extent $\mathbf{4}$, positioning their mono-substituted pyrimidine rings more co-planar with the pyrazine core, leading to reduced occupancy of the S1 $\beta$ subsite. The resulting "downward" positioning of the pyrazine 3-amino group strengthened its hydrogen bond to the side chain hydroxyl of the catalytic Ser195.

Water molecules can play an important stabilising role in protein-ligand complexes ${ }^{84}$ and conserved water molecules are on average more tightly bound than displaced water molecules..$^{85}$ In a previous report, MD simulations and MM/PBSA binding free energy calculations on five guanidine-based uPA inhibitors showed that a water-mediated hydrogen bond network located near the S1 pocket of UPA contributed to inhibitor potency and drove selectivity of the ligands for human uPA over related trypsin-like serine proteases (like tPA). ${ }^{86}$ The water-mediated hydrogen bonds observed here for amiloride, HMA and analogues in their human uPA-bound X-ray cocrystal structures between the carbonyl oxygen of the acylguanidine and the backbone nitrogen and carbonyl of Ser214 and Val227, respectively, were all maintained throughout the MD simulations. 
a

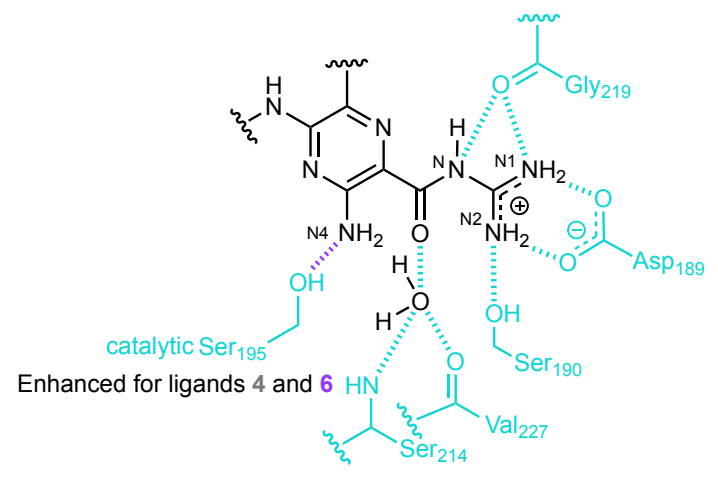

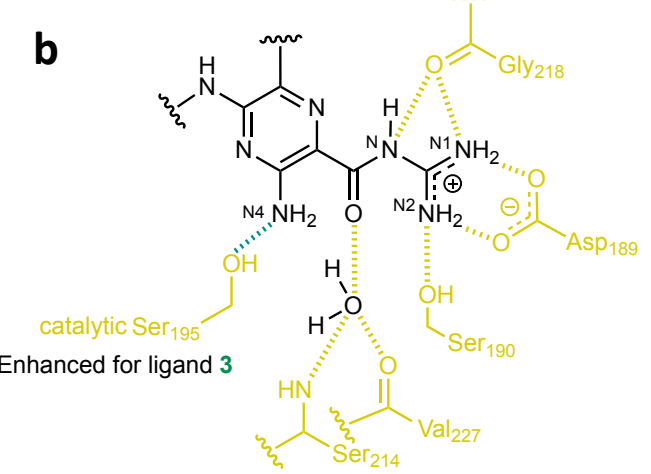

Fig.1 Summary of significant hydrogen bond interactions identified in MD simulations of amiloride 1, HMA 2 and 6-substituted analogues 3-6 complexes with: (a) human and (b) mouse uPA. Hydrogen bonds that were observed in the human X-ray co-crystal structures and preserved in MD simulations in all ligands; e.g. to the Asp189 carboxylate, Ser190 side chain hydroxyl, S1ß Gly218/Gly219 backbone carbonyl and the water-mediated hydrogen bonds to the backbone nitrogen of Ser214 and backbone carbonyl of Val227, are shown in cyan (human UPA) and gold (mouse uPA). The hydrogen bond between the side chain hydroxyl of the catalytic Ser195 and the pyrazine 3-amino group (N4) of ligands 4 (grey) and $\mathbf{6}$ (purple) in human uPA were maintained in the MD simulations (left). The same hydrogen bond was maintained for ligand $\mathbf{3}$ in the mouse UPA MD simulation (shown in green on the right). Snapshots from the MD simulations after 100 ns are provided in Figs. S10 and S11. For detailed analyses refer to Tables S5 (human uPA) and S6 (mouse uPA).

In their interactions with mouse uPA, ligands 1-6 all showed the conserved salt bridge interaction with Asp189. Hydrogen bonds between the two terminal nitrogens of the acylguanidines and the side chain hydroxyl of Ser190 and the backbone nitrogen of the S1 $\beta$ Gly218, which were conserved across the respective docked structures, were also maintained throughout the MD simulations (Fig. S11). The absence of direct hydrogen bond interactions between the ligands and active site residues that vary between the two proteins (i.e. 60, 99, 146 and 192) suggests that uPA human/mouse species selectivity in this class is driven by other factors. It was noted that the water molecule in the S1 pocket was absent in the X-ray structure of mouse uPA. However, during the MD simulations, a water molecule from the bulk moved into the S1 pocket and formed water mediated hydrogen bonds between the ligand carbonyl oxygens and Ser214 and Val227. This finding underscores the importance of water-mediated hydrogen bonding networks for uPA binding in these amiloride-based inhibitors.

Substitution of Gln192 in human uPA to Lys192 in mouse uPA partially contributes to human/mouse species selectivity

A detailed energy-component analysis for ligands 1-6 and their interactions with selected human uPA residues was extracted from the MD simulation trajectories (Table S7). In their mouse UPA complexes, ligands 1-6 all showed favourable interactions with $S 1 \beta$ residue Glu146 but suffered from poor interactions with Lys192. In contrast, interactions with the corresponding residues Ser 146 and GIn192 in the human uPA complexes were both favourable, suggesting that the change to Lys192 contributes, at least partially, to loss of potency against mouse uPA.

Ligand relative binding free energies calculated from MD simulations align with human uPA experimental data
To complement the MD simulations, we alchemically perturbed the ligands to one another and estimated the relative human UPA binding free energies between HMA 2 and 6-substituted analogues 3-6, between ligands 3 and 5 and ligands 5 and 6 (Fig. 2 and Table S9). Energies were calculated using Eq. 1 (see Experimental) using the thermodynamic cycle outlined in Fig. S4. Error analyses were performed using the Bennett acceptance ratio estimator (BAR) ${ }^{73}$ as implemented in ParseFep in VMD 1.9.2.72 Model convergence was monitored by examining the closed thermodynamic cycle (i.e. perturbations from ligands $\mathbf{2}$ to $\mathbf{3}, \mathbf{3}$ to $\mathbf{5}$ and $\mathbf{5}$ to 2 ). The thermodynamic cycle was found to close within $\sim 0.7 \mathrm{kcal} / \mathrm{mol}$ (Fig. S12). The relative binding free energy calculations correctly predicted that human uPA binding affinities improve $(1-2 \mathrm{kcal} / \mathrm{mol})$ following introduction of substituents at the 6-position of HMA 2. The simulations ranked the relative potencies of ligands $\mathbf{3}$ and $\mathbf{5}$ with an error of $0.3 \mathrm{kcal} / \mathrm{mol}$ when compared to the experimentally observed trends (Table S10). The simulation underestimated the binding free energy difference between ligands 2 and 4 by $1.0 \mathrm{kcal} / \mathrm{mol}$ and between ligands 2 and 6 by $1.2 \mathrm{kcal} / \mathrm{mol}$ (Table $\mathrm{S} 10)$. As our convergence check indicated that the FEP simulations converged within $0.7 \mathrm{kcal} / \mathrm{mol}$, it is possible inadequacies in the force field contributed to this discrepancy. ${ }^{87-89}$

Studies with partially murinised human UPA (H99Y) carrying a single residue mutation

To study the effects of a single residue mutation from His99 (present in human uPA) to Tyr (present in mouse uPA), we measured inhibition $\left(K_{\mathrm{i}}\right)$ of the partially murinised human uPA (H99Y) by amiloride 1, HMA 2 and analogues $\mathbf{3}$ and 5. As seen with human versus mouse UPA, amiloride showed only a slight difference in activity between the two enzymes. 


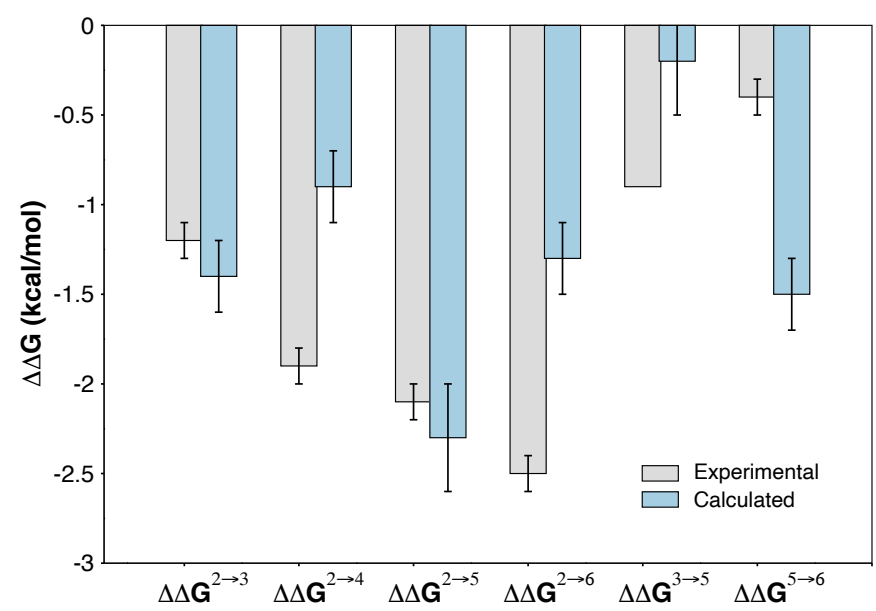

Fig. 2 Experimental and calculated relative binding free energies ( $\mathrm{kcal} / \mathrm{mol}$ ) for perturbations of HMA 2 to 6-substituted analogues 3$\mathbf{6}$, ligand $\mathbf{3}$ to $\mathbf{5}$ and ligand $\mathbf{5}$ to $\mathbf{6}$. (see Table S9 for raw data). Forward and backward simulations were combined using the Bennett acceptance ratio (BAR) estimator. ${ }^{73}$ Error bars correspond to propagated errors.

For HMA, which showed 6.9-fold lower activity against mouse UPA than human uPA (Table 1), a much greater loss of potency (>36.9fold) was observed with H99Y. Dramatically reduced potencies were also seen with $\mathbf{3}$ and $\mathbf{5}$ against H99Y (Table 2), suggesting a key role for residue 99 in the human/mouse uPA species selectivity of HMA 2 and 6-substituted analogues.

To establish whether our in silico models could reproduce the experimentally observed human/H99Y selectivity, we calculated the relative binding free energies arising after perturbation of His99 to Tyr99 in the presence of the four ligands. The FEP calculations predicted a relative selectivity of $-0.1 \mathrm{kcal} / \mathrm{mol}$ for amiloride 1 and 1.0, 1.4 and $1.9 \mathrm{kcal} / \mathrm{mol}$ for HMA 2 and analogues $\mathbf{3}$ and $\mathbf{5}$, respectively (Fig. 3), thus capturing the experimental trends.

Table 2 Inhibitory potencies of amiloride 1, HMA 2 and 6-substituted analogues $\mathbf{3}$ and $\mathbf{5}$ against human uPA and partially murinised human UPA (H99Y).

\begin{tabular}{ccc}
\hline Ligands & $\mathrm{H} 99 Y K_{\mathrm{i}}(\mathrm{nM})^{a}$ & Human/H99Y selectivity factor \\
\hline Amiloride 1 & 5,900 & 2.4 \\
HMA 2 & $>50,000$ & $>36.9$ \\
$\mathbf{3}$ & 8,015 & 43.8 \\
$\mathbf{5}$ & 6,880 & 163.8 \\
\hline
\end{tabular}

${ }_{a}$ Values represent the average of two independent experiments.

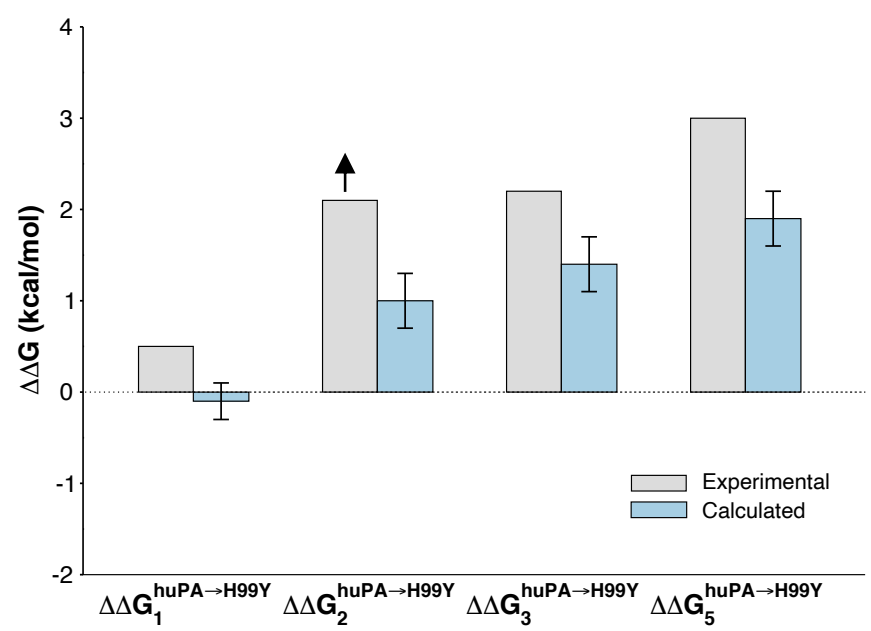

Fig. 3 Experimental and calculated relative binding free energies $(\mathrm{kcal} / \mathrm{mol})$ after mutation of His99 in human uPA to Tyr99 in partially murinised human uPA (H99Y) in the presence of ligands 1, 2, 3 and 5 (see Table S11 for raw data). Error bars correspond to the propagated errors obtained when combining the forward and backward simulations using the Bennett acceptance ratio (BAR) estimator. ${ }^{73}$

Having established an apparent role for residue 99, we sought to understand its molecular basis by solving the X-ray co-crystal structures of H99Y bound to HMA 2 (H99Y-2; PDB 6JYP; $2.25 \AA$ ) , 3 (H99Y-3 PDB 6L05; $2.90 \AA$ ) and 5 (H99Y-5; PDB 6L04; $2.40 \AA$ ) (Table $\mathrm{S} 2)$. With the $\mathrm{X}$-ray structures in hand, $\mathrm{MD}$ simulations were performed on apo-H99Y, the three ligand-bound H99Y complexes and an H99Y-amiloride 1 complex obtained from docking.

RMSD analysis of the protein $C_{a}$ atoms in the apo H99Y and ligand-bound simulations stabilised around 1.0-1.5 $\AA$ (Fig. S13A and $B)$. With the exception of amiloride 1 , the RMSDs of the ligand heavy atoms in the H99Y-ligand complexes showed higher values than in the human uPA complexes, stabilising around 2.0-4.0 $\AA$ (Fig. S13C). This suggests that the stability of amiloride $\mathbf{1}$ in the binding site is less affected by the H99Y mutation than HMA 2 and the 6substituted analogues $\mathbf{3}$ and $\mathbf{5}$.

RMSF of the protein $\mathrm{C}_{a}$ atoms in the apo-H99Y and liganded simulations showed similar patterns to human UPA, indicating that the single residue mutation did not significantly alter protein dynamics (Fig. S13D). Regions of protein flexibility and rigidity were consistent for RMSF values derived from the X-ray B-factors and MD simulations. All ligands displayed reduced flexibility around the S1 and $\mathrm{S} 1 \beta$ pockets (Fig. S13E).

The conserved water molecule observed in the S1 pocket of all human UPA X-ray co-crystal structures and maintained throughout MD simulations (Fig. 1) was absent in the H99Y-2 and H99Y-3 X-ray co-crystal structures. In contrast, the H99Y-5 X-ray structure retained this water molecule. During the simulations, a water molecule from the bulk solvent moved into the S1 pocket to form the conserved hydrogen bond network between the carbonyl oxygen of the acylguanidine of amiloride 1, HMA $\mathbf{2}$ and $\mathbf{3}$ and the backbone carbonyl oxygen of Val227 and the backbone nitrogen of 
Ser214, further supporting the importance of these interactions for ligand binding. In their H99Y complexes, amiloride 1, HMA 2 and analogues $\mathbf{3}$ and $\mathbf{5}$ maintained the hydrogen bonds between their terminal acylguanidine nitrogens and Asp189, Ser190 and Gly219 (Fig. S14 and Table S12). No direct interactions were evident in the simulations between the ligands and residue 99 , as seen in the X-ray structures.

In view of these findings, it remained unclear why HMA $\mathbf{2}$ and its 6-substituted analogues $\mathbf{3}$ and $\mathbf{5}$ showed dramatic potency losses against H99Y relative to human uPA despite: (1) showing similar interactions with both enzymes, (2) mutated residue 99 not being in direct contact with the ligands ( $\sim 7 \AA$ away) and (3) the protein and ligand conformations remaining unchanged. This led us to examine the enthalpic and entropic contributions to protein binding of HMA 2 arising from the single residue mutation. FEP calculations were performed at seven $5 \mathrm{~K}$ interval temperatures ${ }^{90,}{ }^{91}$ between 283.15 and $313.15 \mathrm{~K}$ and the relative changes in enthalpy (slope) and entropy ( $y$-intercept) were extrapolated from the $\Delta \Delta G / T$ versus $1 / T$ plot (Fig. S15). These calculations revealed that the reduction in H99Y binding affinity $(\Delta \Delta \mathrm{G}=1.0 \pm 0.3 \mathrm{kcal} / \mathrm{mol})$ was enthalpically driven $(\Delta \Delta \mathrm{H}=14.8 \mathrm{kcal} / \mathrm{mol})$ and was partially compensated for by an entropy gain $(-\mathrm{T} \Delta \Delta \mathrm{S}=-13.4 \mathrm{kcal} / \mathrm{mol})$. This finding provided the first evidence that reduced $\mathrm{H} 99 \mathrm{Y}$ potency may result from perturbation of the water network in the binding site.

\section{Human/mouse uPA selectivity is due to disruption of the water network}

In a recent report, unfavourable changes in water networks surrounding a ligand in the binding site of Haemophilus influenzae virulence protein SiaP were identified as the cause of dramatic reductions in ligand affinity following a single residue mutation..$^{92}$ The mutated residue (Ala to Asn) was not in close contact with the ligand and the protein and ligand conformations were retained in both complexes. ${ }^{92} \mathrm{~A} \Delta \Delta \mathrm{H}$ value of $12.4 \mathrm{kcal} / \mathrm{mol}$ and $-\mathrm{T} \Delta \Delta \mathrm{S}$ of -8.0 $\mathrm{kcal} / \mathrm{mol}$ were observed. To examine whether a similar effect might be contributing to human/mouse uPA selectivity, we examined the water networks surrounding our ligands, particularly around residue 99, using average water density maps generated from the MD simulations. Fig. 4 shows the density of water molecules surrounding amiloride $\mathbf{1}$ and HMA $\mathbf{2}$ in the human, mouse and partially murinised human UPA (H99Y) active sites. The maps revealed that in human uPA (i.e. His99), both ligands are surrounded by eleven water molecules (W1-W11). In mouse UPA and H99Y (both Tyr99), amiloride maintains the W1-W11 water network. For HMA 2, one water molecule (W9) was lost in the H99Y complex and in mouse uPA, two more water molecules (W7 and W10) were missing.
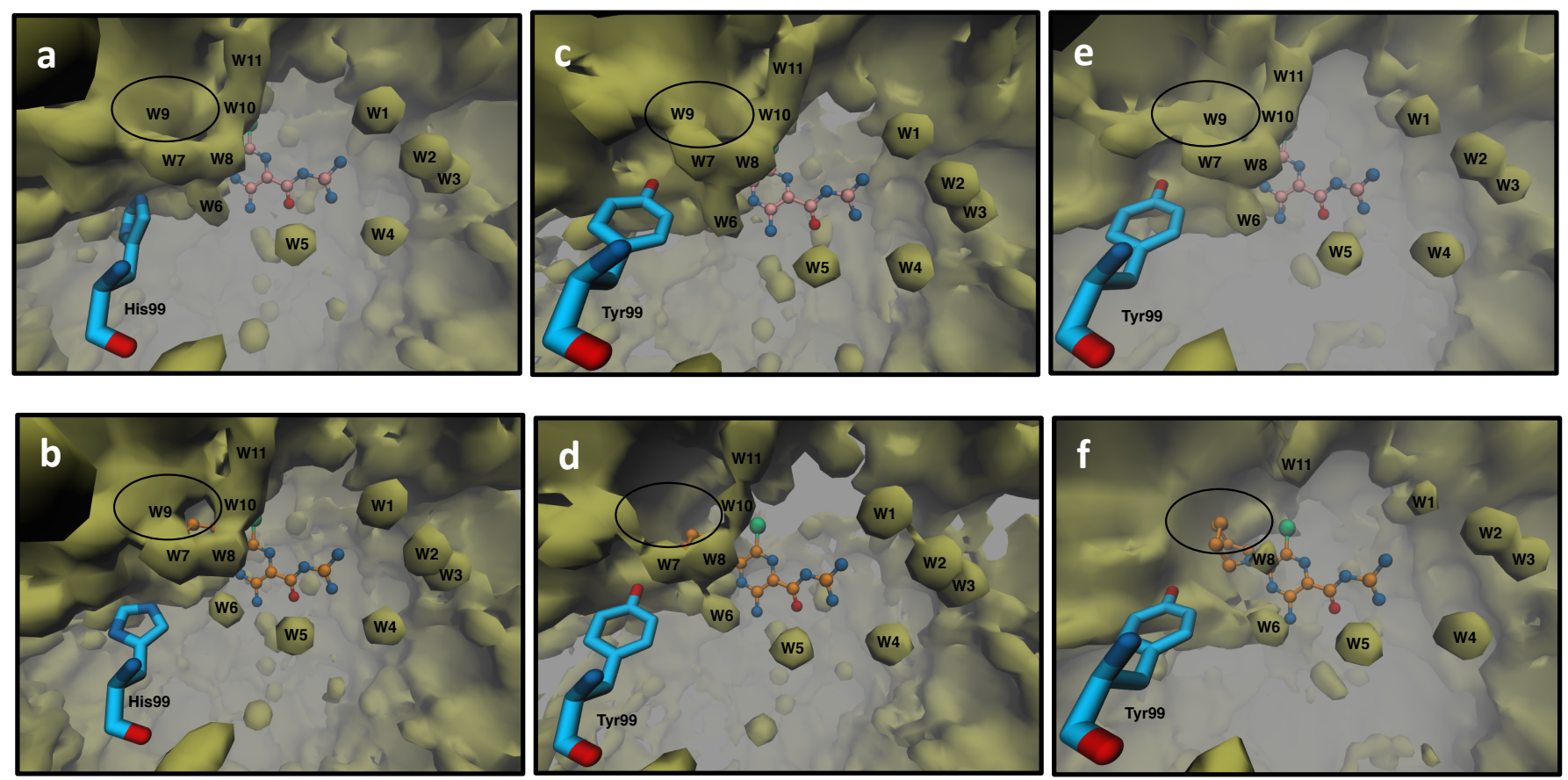

Fig. 4 Maps showing the average water density surrounding amiloride 1 (top panels a, c, e) and HMA 2 (bottom panels b, d, f) when bound to ( $a$ and b) human uPA (His99), ( $c$ and d) partially murinised human uPA (H99Y) and (e and f) mouse uPA (Tyr99). Eleven water molecules surrounding the ligands in the human uPA structure are numbered W1-W11. W5 corresponds to the conserved water molecule in the S1 pocket. Amiloride 1 retains the W1-W11 network in all complexes. HMA 2 loses W9 in its H99Y complex (d) and W7, W9 and W10 are absent in its mouse uPA complex (f). 
Similarly, in their human uPA complexes, ligands 3 and 6 displayed the same water network (W1-W11) as amiloride 1 and HMA 2 (Fig. S16). Ligand 4 retained only five of the eleven water molecules (W5-W9) but showed one new water (W12). Ligand 5 showed ten water molecules (W10 absent). In its H99Y complex, ligand $\mathbf{3}$ lost one water (W9) and in its mouse uPA complex only three water molecules (W2, W3 and W4) remained. For ligand 5, W9, W10 and W11 were lost from the network in the H99Y structure and W7 and W9-W11 were missing in the mouse uPA complex. Ligands 4 and 6 had both lost waters W7-W11 in their mouse uPA complexes. Thus, while amiloride 1 was able to maintain the W1-W11 water network across human, mouse and H99Y complexes, the mouse and H99Y complexes showed lower numbers of water molecules participating in the network for all of the HMA-based ligands. In all of the H99Y complexes, a key water molecule (W9) residing between residue 99 and the ligand $5-N, N$-hexamethylene ring appeared to contribute significantly to the loss of potency (Fig. 4 and Fig. S16).

In the mouse uPA complexes, W9-W11 were always missing (Fig. 4 and Fig. S16), contributing to further losses in potency. Together these data indicate that when residue 99 is Tyr, the 5-N,Nhexamethylene ring at the 5-position of HMA 2 and its 6substituted analogues 3-6 causes expulsion of water molecule (W9) in the H99Y complexes, and additional losses of water molecules in the mouse complexes.

\section{Removal of the $5-\mathrm{N}, \mathrm{N}$-hexamethylene ring improves human/ mouse selectivity}

To test the effect of removing the $5-N, N$-hexamethylene ring on human/mouse uPA selectivity, we selected ligand $\mathbf{7}$ for further study. Compound 7 represents a direct analogue of 4 lacking the $5-\mathrm{N}, \mathrm{N}$ hexamethylene ring. Compound $\mathbf{4}$ is a potent and selective human UPA inhibitor that inhibits liver metastases in a mouse model of pancreatic cancer. ${ }^{41}$ An X-ray co-crystal structure of $\mathbf{7}$ bound to human uPA solved previously (PDB 6AG2; $1.8 \AA)^{30}$ and MD simulations of the complex performed here showed that the RMSD of the protein $C_{a}$ atoms stabilised around 1.0-1.5 $\AA$ and the ligand heavy atom RMSD around 1.0-3.0 $\AA$ (Fig. S17). Similar to ligands 1-6, higher protein $C_{a}$ atom and ligand RMSD values were observed in the mouse uPA complex compared to the human uPA complex. RMSF of protein $C_{a}$ atoms in the presence of ligand 7 displayed similar fluctuations as ligands 1-6 in their human and mouse uPA complexes (Fig. S17). Reduced protein flexibility was observed at the S1 and S1 $\beta$ pockets where the ligand interacts with the proteins. The simulations showed that in both human and mouse uPA complexes, ligand 7 maintains the conserved interactions with Asp189, Gly219 and Ser190 and water-mediated hydrogen bonds with Ser214 and Val227 (Fig. 5).

Interestingly, ligand $\mathbf{7}$ was the only compound that maintained the hydrogen bond between the nitrogen atom of the pyrazine 3-amino group and the side chain hydroxyl of Ser195 in both human and mouse MD simulations (Fig. 5). The potency of $\mathbf{7}$ against human uPA was reported at $K_{\mathrm{i}}=204 \pm 14 \mathrm{nM} .{ }^{30}$ Here, we determined that 7 shows $K_{\mathrm{i}}=956 \mathrm{nM}$ against mouse uPA, thus showing reduced selectivity for the human enzyme (selectivity factor $=4.7$, Table S17) relative to HMA and its analogues. Importantly, as observed for amiloride 1, water density maps showed that ligand $\mathbf{7}$ maintains a similar water network in both human and mouse uPA complexes (Fig. 5).

\section{Conclusions}

The primary aim of this study was to identify the molecular basis for the human/mouse species selectivity observed with 6-substituted 5$\mathrm{N}, \mathrm{N}$-(hexamethylene)amiloride-based uPA inhibitors. While the parent drug amiloride shows similar potency against both enzymes, the substituted derivatives, which have higher human uPA potency and potential for development as uPA-targeting anti-metastasis drugs, are significantly less potent against the mouse variant, thus confounding interpretation of data when tested in mouse cancer models. The inability of initial docking studies to faithfully reproduce the experimentally determined potencies of the inhibitors against human and mouse uPA led us to explore other computational methods in search of an explanation. Comprehensive MD simulations revealed remarkably similar binding poses, hydrogen bonding patterns and other interactions for the ligands when complexed to the two enzymes but no obvious drivers of affinity differences were evident, although the change from Gln192 in human uPA to Lys192 in mouse UPA appeared to make a small contribution. Free energy perturbation calculations that recapitulated the experimentally determined inhibitory potencies validated our modelling and analysis.

Detailed examination of the effect of changing residue 99 from His in human uPA to Tyr in mouse uPA proved more fruitful. X-ray co-crystal structures of selected ligands bound to partially murinised human UPA (H99Y) in combination with FEP calculations hinted that water networks around this residue may play an important role in selectivity. Further analysis revealed that loss of a single water (W9) close to the 5-N,N-hexamethylene ring of HMA 2 and its analogues causes an enthalpy-driven loss of potency. This finding was confirmed when compound 7, a direct analogue of $\mathbf{4}$ lacking the 5$\mathrm{N}, \mathrm{N}$-hexamethylene ring, was shown to maintain a similar water network when bound to both enzymes and did not suffer the same dramatic loss of potency as other derivatives. Overall, the study highlights the crucial role that binding site water molecules can play in the affinity of ligands for their protein targets and provides a framework for the development of next-generation amiloride-based inhibitors that can better report uPA target-based activity in mouse cancer models.

\section{Author Contributions}

N.S.E performed the MD simulations, free energy calculations and analysed all data under the direction of H.Y and M.J.K. B.J.B performed the enzyme activity assays. L.J solved all X-ray crystal structures. All authors contributed to drafting and revision of the manuscript. 


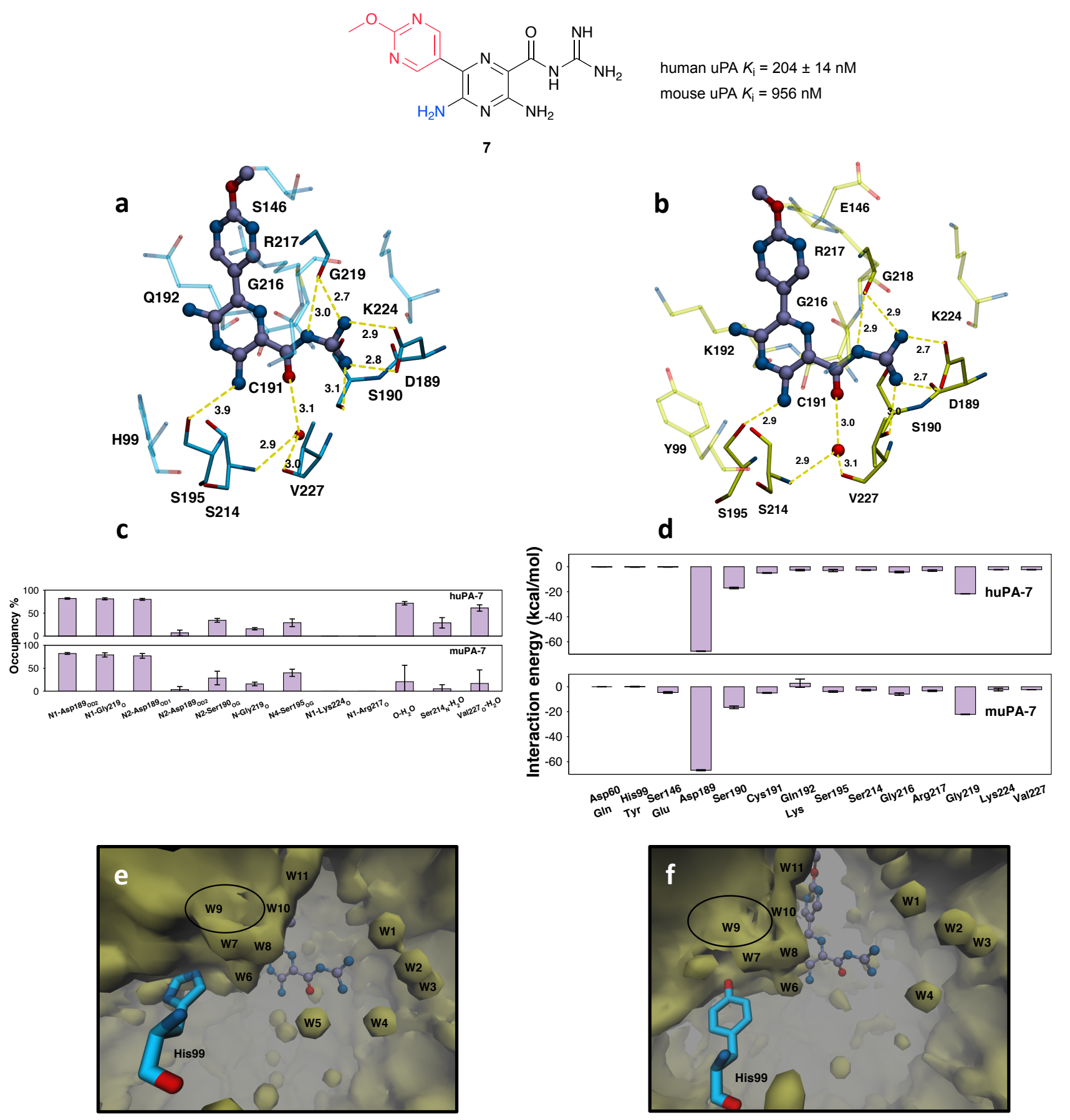

Fig. 5 Snapshots from MD simulations of ligand 7 (iceblue) bound to: (a) human (cyan) and (b) mouse (gold) uPA. (c) Hydrogen bond interactions. Hydrogen bonds that were conserved in the $X$ ray co-crystal structures and maintained in MD simulations in all ligands; i.e. Asp189 carboxylate, Ser190 side chain hydroxyl, S1ß Gly218/Gly219 backbone carbonyl and water-mediated hydrogen bonds to the backbone nitrogen of Ser214 and backbone carbonyl of Val227, were present for $\mathbf{7}$. Ligand $\mathbf{7}$ also showed a hydrogen bond to the side chain hydroxyl of Ser195 in both human and mouse uPA. (d) Interaction energies of ligand 7 with selected residues of human and mouse uPA. Error reported is the standard deviation. For detailed analyses, refer to Tables S15 and S16. Maps showing the average water density surrounding ligand 7 (iceblue) when bound to (e) human uPA (His99) and (f) mouse uPA (Tyr99) uPA. Eleven water molecules surrounding the ligands at the binding sites are numbered W1-W11. W5 is missing in the mouse complex. 


\section{Conflicts of interest}

There are no conflicts to declare.

\section{Acknowledgements}

This work was funded by an Australian National Health and Medical Research Council (NHMRC) Project Grant (APP1100432) awarded to M.K., M.R. and M.H. and a UOW SMAH Small Research Grant (H.Y.). B.J.B. gratefully acknowledges salary support from the Illawarra Cancer Carers. The research was undertaken using resources at the $\mathrm{NCl}$ National Facility systems at the Australian National University, provided through the Australian Government National Computational Merit Allocation Scheme (project ID v15).

\section{Accession Codes}

Coordinates for the partially murinised human uPA (H99Y) inhibitor complexes H99Y-2 (PDB 6JYP; $2.25 \AA ̊$ ), H99Y-3 (PDB 6L05; $2.9 \AA$ ) and H99Y-5 (PDB 6L04; $2.4 \AA$ ) have been deposited in the RSCB PDB.

\section{References}

1. P. A. Andreasen, R. Egelund and H. H. Petersen, Cell. Mol. Life Sci., 2000, 57, 25-40.

2. S. Ulisse, E. Baldini, S. Sorrenti and M. D'Armiento, Curr. Cancer Drug Targets, 2009, 9, 32-71.

3. A. Salajegheh, Urokinase Plasminogen Activator. In: Angiogenesis in Health, Disease and Malignancy., Springer, 2016.

4. D. R. Croucher, D. N. Saunders, S. Lobov and M. Ranson, Nat. Rev. Cancer, 2008, 8, 535.

5. M. J. Duffy and C. Duggan, Clin. Biochem., 2004, 37, 541-548.

6. J. D. Vassalli, A. P. Sappino and D. Belin, J. Clin. Invest., 1991, 88, 1067-1072.

7. S. Strickland and W. H. Beers, J. Biol. Chem., 1976, 251, 56945702.

8. K. Ogiwara, A. Hagiwara, S. Rajapakse and T. Takahashi, Biol. Reprod., 2015, 92, 10, 1-17.

9. F. Khamsi, D. T. Armstrong and X. Zhang, Mol. Hum. Reprod., 1996, 2, 273-276.

10. M. H. Weng, C. J. Chang, W. Y. Chen, W. K. Chou, H. C. Peh, M. C. Huang, M. T. Chen and H. Nagahata, J. Dairy Sci., 2006, 89, 20252037.

11. J. M. Beck, A. M. Preston and M. R. Gyetko, Infect. Immun., 1999, 67, 879-884.

12. P. J. Declerck, H. R. Lijnen, M. Verstreken, H. Moreau and D. Collen, Blood, 1990, 75, 1794-1800.

13. R. Pannell, S. Li and V. Gurewich, J. Thromb. Thrombolysis, 2017, 44, 210-215.

14. B. J. Buckley, U. Ali, M. J. Kelso and M. Ranson, Curr. Drug Targets, 2019, 20, 970-981.

15. J. A. Hamilton, Arthritis Rheum., 2008, 58, 645-648.

16. N. Toki, H. Tsushima, M. Yamasaki, R. Yamasaki and T. Yamura, J. Invest. Dermatol., 1982, 78, 18-23.

17. R. Miskin and R. Ben-Ishai, PNAS, 1981, 78, 6236-6240.

18. D. Gur-Wahnon, T. Mizrachi, F.-Y. Maaravi-Pinto, A. Lourbopoulos, N. Grigoriadis, A. A. R. Higazi and T. Brenner, J. Neuroinflammation, 2013, 10, 1-11.
19. V. Stepanova, K. V. Dergilev, K. R. Holman, Y. V. Parfyonova, Z. I. Tsokolaeva, M. Teter, E. N. Atochina-Vasserman, A. Volgina, S. V. Zaitsev, S. P. Lewis, F. G. Zabozlaev, K. Obraztsova, V. P. Krymskaya and D. B. Cines, J. Biol. Chem., 2017, 292, 2052820543.

20. L. Raij, R. Tian, J. S. Wong, J. C. He and K. N. Campbell, Am. J. Physiol. Renal Physiol., 2016, 311, F1308-f1317.

21. A. H. Mekkawy, M. H. Pourgholami and D. L. Morris, Med. Res. Rev., 2014, 34, 918-956.

22. T. Kaneko, H. Konno, M. Baba, T. Tanaka and S. Nakamura, Cancer Sci., 2003, 94, 43-49.

23. D. Brungs, J. Chen, M. Aghmesheh, K. L. Vine, T. M. Becker, M. G. Carolan and M. Ranson, Oncotarget, 2017, 8, 23099-23109.

24. M. E. L. v. d. Burg, S. C. Henzen-Logmans, E. M. J. J. Berns, W. L. J. v. Putten, J. G. M. Klijn and J. A. Foekens, Int. J. Cancer, 1996, 69, 475-479.

25. N. L. E. Harris, C. Vennin, J. R. W. Conway, K. L. Vine, M. Pinese, M. J. Cowley, R. F. Shearer, M. C. Lucas, D. Herrmann, A. H. Allam, M. Pajic, J. P. Morton, I. Australian Pancreatic Cancer Genome, A. V. Biankin, M. Ranson, P. Timpson and D. N. Saunders, Oncogene, 2017, 36, 4288-4298.

26. L. Tang and X. Han, Biomed. Pharmacother., 2013, 67, 179-182.

27. M. J. Duffy, P. O'Grady, D. Devaney, L. O'Siorain, J. J. Fennelly and H. J. Lijnen, Cancer, 1988, 62, 531-533.

28. L. Harris, H. Fritsche, R. Mennel, L. Norton, P. Ravdin, S. Taube, M. R. Somerfield, D. F. Hayes and R. C. Bast Jr, J. Clin. Oncol., 2007, 25, 5287-5312.

29. I. Henneke, S. Greschus, R. Savai, M. Korfei, P. Markart, P. Mahavadi, R. T. Schermuly, M. Wygrecka, J. Stürzebecher, W. Seeger, A. Günther and C. Ruppert, Am. J. Respir. Crit. Care Med., 2010, 181, 611-619.

30. B. J. Buckley, H. Majed, A. Aboelela, E. Minaei, L. Jiang, K. Fildes, C.-Y. Cheung, D. Johnson, D. Bachovchin and G. M. Cook, Bioorg. Med. Chem. Lett., 2019, 29, 126753.

31. J. Rømer, C. Pyke, L. R. Lund, J. Eriksen, P. Kristensen, E. Rønne, G. Høyer-Hansen, K. Danø and N. Brünner, Int. J. Cancer, 1994, 57, 553-560.

32. R. Hildenbrand, A. Schaaf, A. Dorn-Beineke, H. Allgayer, M. Sütterlin, A. Marx and P. Stroebel, Histol. Histopathol., 2009, 24, 869-877.

33. J. Rømer, C. Pyke, L. R. Lund, J. Eriksen, P. Kristensen, E. Rønne, G. Høyer-Hansen, K. Danø and N. Brünner, Int. J. Cancer, 1994, 57, 553-560.

34. T. L. Frandsen, C. Holst-Hansen, B. S. Nielsen, I. J. Christensen, J. R. Nyengaard, P. Carmeliet and N. Brünner, Cancer Res., 2001, 61, 532-537.

35. M. Ploug, S. Ostergaard, H. Gardsvoll, K. Kovalski, C. HolstHansen, A. Holm, L. Ossowski and K. Dano, Biochemistry, 2001, 40, 12157-68.

36. V. Hofmeister, D. Schrama and J. C. Becker, Cancer Immunol. Immunother., 2008, 57, 1-17.

37. H. Matthews, M. Ranson and M. J. Kelso, Int. J. Cancer, 2011, 129, 2051-2061.

38. J. D. Vassalli and D. Belin, FEBS Lett., 1987, 214, 187-191.

39. E. Zeslawska, A. Schweinitz, A. Karcher, P. Sondermann, S. Sperl, J. Stürzebecher and U. Jacob, J. Mol. Biol., 2000, 301, 465-475.

40. P. Palsgaard, F. A. Gorin and I. Vorobyov, Biophys. J., 2018, 114, $56 a$.

41. B. J. Buckley, A. Aboelela, E. Minaei, L. X. Jiang, Z. Xu, U. Ali, K. Fildes, C.-Y. Cheung, S. M. Cook, D. C. Johnson, D. A. Bachovchin, G. M. Cook, M. Apte, M. Huang, M. Ranson and M. J. Kelso, J. Med. Chem., 2018, 61, 8299-8320. 
42. C. W. Song, J. C. Lyons, C. M. Makepeace, R. J. Griffin and E. J. Cragoe Jr, Int. J. Radiat. Oncol. Biol. Phys., 1994, 30, 133-139.

43. J. Luo and I. Tannock, Br. J. Cancer, 1994, 70, 617.

44. A. R. Rowson-Hodel, A. L. Berg, J. H. Wald, J. Hatakeyama, K. VanderVorst, D. A. Curiel, L. J. Leon, C. Sweeney and K. L. Carraway III, Cancer Lett., 2016, 375, 62-72.

45. V. L. Nienaber, D. Davidson, R. Edalji, V. L. Giranda, V. Klinghofer, J. Henkin, P. Magdalinos, R. Mantei, S. Merrick, J. M. Severin, R. A. Smith, K. Stewart, K. Walter, J. Wang, M. Wendt, M. Weitzberg, X. Zhao and T. Rockway, Structure, 2000, 8, 553-63.

46. B. A. Katz, R. Mackman, C. Luong, K. Radika, A. Martelli, P. A. Sprengeler, J. Wang, H. Chan and L. Wong, Chem. Biol., 2000, 7, 299-312.

47. R. L. Mackman, B. A. Katz, J. G. Breitenbucher, H. C. Hui, E. Verner, C. Luong, L. Liu and P. A. Sprengeler, J. Med. Chem., 2001, 44, 3856-3871.

48. B. A. Katz, P. A. Sprengeler, C. Luong, E. Verner, K. Elrod, M. Kirtley, J. Janc, J. R. Spencer, J. G. Breitenbucher and H. Hui, Chem. Biol., 2001, 8, 1107-1121.

49. V. Klinghofer, K. Stewart, T. McGonigal, R. Smith, A. Sarthy, V. Nienaber, C. Butler, S. Dorwin, P. Richardson, M. Weitzberg, M. Wendt, T. Rockway, X. Zhao, K. I. Hulkower and V. L. Giranda, Biochemistry, 2001, 40, 9125-9131.

50. A. Schweinitz, T. Steinmetzer, I. J. Banke, M. J. E. Arlt, A. Stürzebecher, O. Schuster, A. Geissler, H. Giersiefen, E. Zeslawska, U. Jacob, A. Krüger and J. Stürzebecher, J. Biol. Chem., 2004, 279, 33613-33622.

51. O. Trott and A. J. Olson, J. Comput. Chem., 2010, 31, 455-461.

52. M. D. Hanwell, D. E. Curtis, D. C. Lonie, T. Vandermeersch, E. Zurek and G. R. Hutchison, J. Cheminform., 2012, 4, 17.

53. M. Sanner, J. Mol. Graph. Model., 1999, 17, 57-61.

54. J. C. Phillips, R. Braun, W. Wang, J. Gumbart, E. Tajkhorshid, E. Villa, C. Chipot, R. D. Skeel, L. Kalé and K. Schulten, J. Comput. Chem., 2005, 26, 1781-1802.

55. W. Humphrey, A. Dalke and K. Schulten, J. Mol. Graph., 1996, 14, 33-38.

56. J. A. Maier, C. Martinez, K. Kasavajhala, L. Wickstrom, K. E. Hauser and C. Simmerling, J. Chem. Theory Comput., 2015, 11, 3696-3713.

57. L. F. Song, T.-S. Lee, C. Zhu, D. M. York and K. M. Merz, J. Chem. Inf. Model., 2019, 59, 3128-3135.

58. W. L. Jorgensen, J. Chandrasekhar, J. D. Madura, R. W. Impey and M. L. Klein, J. Chem. Phys., 1983, 79, 926-935.

59. J. Wang, W. Wang, P. A. Kollman and D. A. Case, J. Mol. Graph. Model., 2006, 25, 247-260.

60. J. Wang, R. M. Wolf, J. W. Caldwell, P. A. Kollman and D. A. Case, J. Comput. Chem., 2004, 25, 1157-1174.

61. A. Jakalian, D. B. Jack and C. I. Bayly, J. Comput. Chem., 2002, 23, 1623-41.

62. M. H. M. Olsson, C. R. Søndergaard, M. Rostkowski and J. H. Jensen, J. Chem. Theory Comput., 2011, 7, 525-537.

63. S. E. Feller, Y. Zhang, R. W. Pastor and B. R. Brooks, J. Chem. Phys., 1995, 103, 4613-4621.

64. S. Miyamoto and P. A. Kollman, J. Comput. Chem., 1992, 13, 952 962.

65. T. Darden, D. York and L. Pedersen, J. Chem. Phys., 1993, 98, 10089-10092.

66. A. Kuzmanic and B. Zagrovic, Biophys. J., 2010, 98, 861-871.
67. J. D. Durrant and J. A. McCammon, J. Mol. Graph. Model., 2011, 31, 5-9.

68. R. W. Zwanzig, J. Chem. Phys., 1954, 22, 1420-1426.

69. A. de Ruiter and C. Oostenbrink, Curr. Opin. Struct. Biol., 2020, 61, 207-212.

70. T. C. Beutler, A. E. Mark, R. C. van Schaik, P. R. Gerber and W. F. van Gunsteren, Chem. Phys. Lett., 1994, 222, 529-539.

71. W. Jespers, M. Esguerra, J. Åqvist and H. Gutiérrez-de-Terán, J. Cheminform., 2019, 11, 26.

72. P. Liu, F. Dehez, W. Cai and C. Chipot, J. Chem. Theory Comput., 2012, 8, 2606-2616.

73. C. H. Bennett, J. Comput. Phys., 1976, 22, 245-268.

74. P. Matricon, A. Ranganathan, E. Warnick, Z.-G. Gao, A. Rudling, C. Lambertucci, G. Marucci, A. Ezzati, M. Jaiteh and D. Dal Ben, Scientific reports, 2017, 7, 1-12.

75. J. Carlsson and J. Åqvist, J. Phys. Chem. B, 2009, 113, 1025510260.

76. B. Zhao, P. Xu, L. Jiang, B. Paaske, T. Kromann-Hansen, J. K. Jensen, H. P. Sorensen, Z. Liu, J. T. Nielsen, A. Christensen, M. Hosseini, K. K. Sorensen, N. C. Nielsen, K. J. Jensen, M. Huang and P. A. Andreasen, PLoS One, 2014, 9, e115872.

77. C. Yung-Chi and W. H. Prusoff, Biochem. Pharmacol., 1973, 22, 3099-3108.

78. G. Zhao, C. Yuan, T. Wind, Z. Huang, P. A. Andreasen and M. Huang, J. Struct. Biol., 2007, 160, 1-10.

79. Z. Otwinowski and W. Minor, Methods Enzymol., 1997, 276, 307326.

80. C. P. Collaborative, Acta Crystallogr. Sect. D. Biol. Crystallogr., 1994, 50, 760.

81. L. Jiang, K. A. Botkjaer, L. M. Andersen, C. Yuan, P. A. Andreasen and M. Huang, Biochem. J., 2013, 449, 161-166.

82. P. Emsley and K. Cowtan, Acta Crystallogr. Sect. D. Biol. Crystallogr., 2004, 60, 2126-2132.

83. T. Kromann-Hansen, E. L. Lange, H. P. Sørensen, G. HassanzadehGhassabeh, M. Huang, J. K. Jensen, S. Muyldermans, P. J. Declerck, E. A. Komives and P. A. Andreasen, Scientific reports, 2017, 7, 3385.

84. J. E. Ladbury, Chem. Biol., 1996, 3, 973-980.

85. C. Barillari, J. Taylor, R. Viner and J. W. Essex, J. Am. Chem. Soc., 2007, 129, 2577-2587.

86. R. Sa, L. Fang, M. Huang, Q. Li, Y. Wei and K. Wu, J. Phys. Chem. A, 2014, 118, 9113-9119.

87. B. J. Williams-Noonan, E. Yuriev and D. K. Chalmers, J. Med. Chem., 2018, 61, 638-649.

88. V. S. Inakollu, D. P. Geerke, C. N. Rowley and H. Yu, Curr. Opin. Struct. Biol., 2020, 61, 182-190.

89. D. Mondal, J. Florian and A. Warshel, J Phys Chem B, 2019, 123, 8910-8915.

90. P. Matricon, R. R. Suresh, Z.-G. Gao, N. Panel, K. A. Jacobson and J. Carlsson, Chem. Sci., 2021, 12, 960-968.

91. A. P. Montgomery, D. Skropeta and H. Yu, Scientific Reports, 2017, 7, 14428.

92. J. F. Darby, A. P. Hopkins, S. Shimizu, S. M. Roberts, J. A. Brannigan, J. P. Turkenburg, G. H. Thomas, R. E. Hubbard and M. Fischer, J. Am. Chem. Soc., 2019, 141, 15818-15826. 